\title{
Phone Calls from the Dead? Exploring the Role of the Trickster
}

Des coups de fil de l'AU-DELÀ? Enquête sur un phénomène paranormal incroyable: Sa folie, ses caractéristiques et son explication by Laurent Kasprowicz. 2018. 136 pp. $€ 20$ (paperback). ISBN 978-2954406237. Available from the author's website. https://www.descoupsdefil.fr/

\section{Reviewed by Sharon Hewitt Rawlette}

sharon.rawlette@gmail.com

https://doi.org/10.31275/2019/1647

Copyright: Creative Commons CC-BY-NC

Even parapsychologists have boggle thresholds. Even those of us who accept the reality of apparitions of the dead and various psychokinetic phenomena such as raps and abnormally blinking lights can have a harder time believing in the reality of phone calls from the dead: that is, cases in which a deceased person speaks with the living via telephone. For one thing, these conversations just seem too straightforward. Typically, in these cases, the voice of the deceased manifests and speaks a message of a few words to several sentences, and in rare cases the voice carries on an extended conversation with a living interlocutor. Those who have experimented with electronic voice phenomena (EVP) know how difficult it is to clearly discern in these communications single words, let alone phrases or the identity of the speaker. And yet, in many cases of supposed phone calls from the dead, the dead seem to speak as clearly as if they were in a living human body at the other end of the telephone line. Is this phenomenon real? And if so, are the dead truly behind it?

Unfortunately, even a century after reports of this phenomenon 
first began, the literature on it remains scant and difficult to come by. There have been two notable English-language books on the topic-D. Scott Rogo and Raymond Bayless's Phone Calls from the Dead (1979) and Callum Cooper's Telephone Calls from the Dead (2012) - but even used copies of these volumes cost upward of $\$ 50$. Nevertheless, interest in this phenomenon does seem to be increasing, as just last year (only six years after the publication of Cooper's book) in March 2018 French sociologist Laurent Kasprowicz published his volume Des coups de fil de l'AU-DELÀ? The title translates to Phone Calls from the BEYOND? Note the question mark. This book seriously considers what is behind these apparent phone calls from the dead and is not willing to settle for easy answers. While Kasprowicz's book is currently available only in French, I hope it will not be long before it is translated into English, as it is an excellent addition to the postmortem phone call literature. It not only presents 17 new cases collected by Kasprowicz but also offers some new, thought-provoking theoretical considerations as well.

Since there is no translation of this book currently available, I will provide here a fairly detailed account of the book's contents, as an aid to English-speaking researchers who may be wondering whether it is worth their while to attempt to read the book in French. I hope they will conclude that it is.

Kasprowicz's book begins with the case that drew him into his study of postmortem telephone communication, an experience that happened to him personally several years ago. While this particular case has aspects that are highly unusual within this genre of experiences, these unusual characteristics are extremely important in that they serve as some of the clues that have led Kasprowicz to his innovative theoretical understanding of the phenomenon.

Kasprowicz's acquaintance with the phenomenon of postmortem telephone communications began four or five days after the death of a beloved dog of his. He was in the car with his mother and brother when he said to them, out loud, "Au paradis, j'espère qu'on y retrouve nos chiens. J'aimerais bien recevoir un signe." (In heaven, I hope we meet our dogs again. I really wish I could get a sign.) One minute later, he received a text message from someone he didn't know. The message read, "SALUT J'AI BIEN REUSSI MON INTEGRATION, TOUT SE PASSE BIEN, JULIE." This translates approximately to, "HI MY ARRIVAL WENT 
GREAT, EVERYTHING'S GOING WELL, JULIE.”

The French word 'intégration,' which I've translated here as "arrival," is used to refer specifically to one's arrival within a new institution or group, like a school or a business enterprise. Kasprowicz received this text at the time of year when school was starting for the fall, so no doubt someone named Julie had been texting someone to let them know that her arrival at her new school was going well. However, Kasprowicz did not know anyone named Julie-somehow her text had been misdirected. And, what was more, the message in the text made complete sense as a response to Kasprowicz's request for a sign that his dog was okay. His dog, first of all, was female (though not named Julie), and it would make perfect sense for a soul who had just reached the "other side" to respond to an inquiry about how they were faring by using the same phrases that Julie used: "Hi my arrival went great, everything's going well."

But that text was only the beginning. The very next day, the Kasprowicz family's landline telephone began to ring-over and over, day and night. When they picked up, however, no one was there. And the information hotline they consulted reported, every time, that no one had called their phone. Other events also suggested that something was acting directly on their telephone and not through the telephone lines. For instance, sometimes their apartment intercom would sound at the same time that the telephone rang. And, on two occasions, the answering machine spontaneously began recording sometime after they had picked up the phone (which it did not normally do). The message the machine recorded in both cases was the sound of jovial laughter, like a family having a good time together around the dinner table.

The following day, something even stranger happened. When the phone rang for the umpteenth time, the family answered it, and after at first hearing only silence, they then heard a knocking or rapping sound, which had a Morse code-like flavor. Kasprowicz says he quickly figured out that one rap stood for no and two raps stood for yes, though he was apparently unacquainted at the time with the prevalence of this code in poltergeist cases. He was then able to ask questions and received the correct responses-even when, to verify that no one was playing a prank on him, he asked "trick" questions: questions that no one except the people in the room should have been able to answer correctly. 
At one point, Kasprowicz posed the question, "Ma chienne était trop vieille?" (Was my dog too old?) A single rap responded "no." This, apparently, was the correct response, as Kasprowicz's dog had not died of old age but of cancer. And then, suddenly, over the telephone came the sound of a dog breathing heavily. This unnerved Kasprowicz, because his dog had specifically died of lung cancer. One hour later, the phone rang again, and again there was a "conversation" with the raps, accompanied

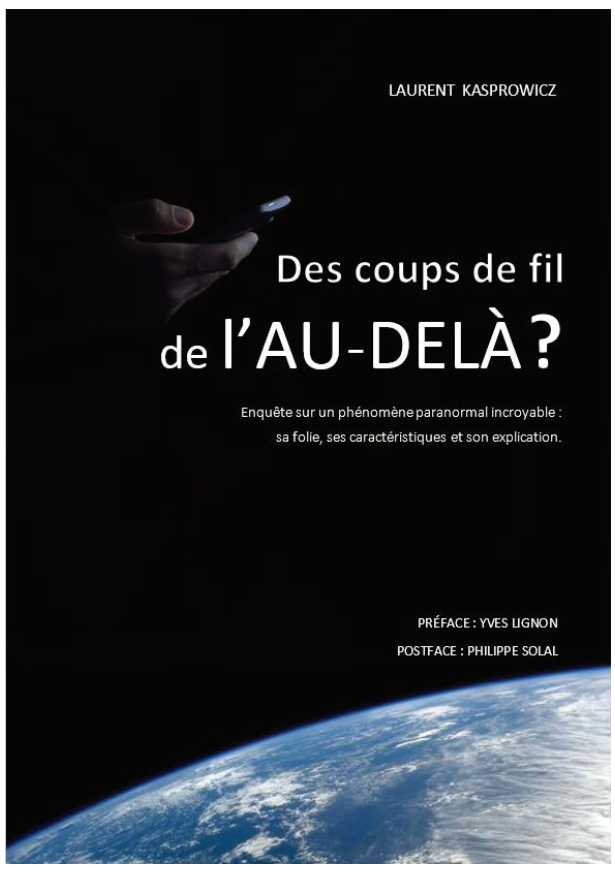
by the sound of the heavy breathing of a dog. Then it cut off, and that was the end of the phenomena.

This experience sparked a strong desire in Kasprowicz to understand what had taken place. In addition to reading the existing books on the topic of phone calls from the dead, Kasprowicz began questioning people in the region where he lived-in northeast France close to the Belgian border-and quickly discovered that apparent telephone communications from the dead were far from uncommon, though all of the other cases Kasprowicz collected involve deceased humans, not animals.

Kasprowicz ended up collecting 17 new cases, including two of his own, and all but two of the cases are from France or neighboring Belgium. Most of them involve spoken messages, either during a "live" call or in a voice message, but his collection also includes some cases in which the phone simply rings anomalously or in which it is answered but no one is on the other end of the line. Two of his cases involve communication through text message, and one involves an apparent two-way conversation with the deceased via computer-based 
instant messaging (the only case that is not technically a telephone communication).

Kasprowicz's book carefully details each of these 17 cases, noting similarities among them as well as unique features. It is important to note that none of the individuals Kasprowicz interviewed were aware of this phenomenon before they themselves experienced it. They certainly were not expecting it to happen and would appear to have had no preconceptions about how it should happen. Nevertheless, Kasprowicz points out several important continuities between these cases and those reported by Rogo and Bayless, and by Cooper, many of which Kasprowicz also describes in detail in his book. Kasprowicz notes the recurrence of the following features:

the association of the phone call with some sort of coincidence or synchronicity (for instance, receiving the phone call on the anniversary and/or at the exact clock time of the death),

hearing breathing or static on the line and/or a metallic or distant voice,

the call occurring on the phone of a friend or relative rather than on one's own phone,

the call apparently coming from a cell phone that is turned off and/or out of battery,

the inability of a telephone operator to trace the call,

the leaving of voice messages directly, without a call having been made,

the offering of reassurances or the delivery of warnings,

the use of certain phrases or some other feature making a clear identification with the deceased, and

the presence of some aspect of the call that seems not quite right-this feature being one that ultimately came to have great theoretical importance for Kasprowicz. 
I would add that, in the cases of seemingly paranormal phone calls that I came across in researching my book The Source and Significance of Coincidences (2019), I found many of these same patterns, though at the time I collected them I had not yet read Kasprowicz's book, nor Rogo and Bayless's or Cooper's.

However, if the similarities across cases are impressive, equally intriguing are the cases collected by Kasprowicz bearing features that are rare or even unique. For instance, there's the case of Nicolas, who got a call from a man who said he was in a coma in a hospital in Warsaw, Poland, and asked Nicolas to say good-bye to his daughter Manue for him. Manue was Nicolas's neighbor and was currently away in Poland with her family. When Manue returned a week later, without knowing anything about the phone call Nicolas had received, she informed Nicolas that her father had been in a coma and died while she was in Poland.

Then there's the case of a woman whose son had recently survived a tragic car accident in which a friend of his died. Two weeks after the death, the mother got a phone call from someone she recognized as her son's deceased friend. The friend seemed panicky and was asking to speak to her son. He said something to the effect of, "Hurry, put him on, please, I don't understand what's going on. It's like the world is turning bizarre. ..."

Also odd is the case of Séverine, who, three months after the death of her mother, was told by her "belle-mère" (either mother-in-law or stepmother) that there was a message for her on the belle-mère's answering machine. She went to listen to it, and it said, "Séverine, Séverine, the Sarah is with me, I'm fine, don't worry. Séverine, Séverine. I'm here. We're doing fine here" (my translation). Sarah was Séverine's family's deceased dog. This would seem to confirm that the caller was Séverine's deceased mother. The strange bit, however, is that Séverine didn't recognize her mother's voice. The sound quality of the recording was good, she said, but it just didn't sound like her mother. This is one of those cases where there's an aspect of the call that is just not quite right- "le détail qui cloche," as Kasprowicz calls it.

I would venture to say, however, that the strangest of the 17 cases Kasprowicz has collected is his own experience after the death of his dog, which I described above. It is strange, first of all, because the 
primary deceased is an animal rather than a human being, and the animal seems to manifest its "voice" in the heavy canine breathing heard over the phone line. But it is also very different from the other cases in Kasprowicz's book because of the way it involves communication by way of yes/no questions answered by raps heard through the phone.

In fact, at the time I read Kasprowicz's book, I thought Kasprowicz's case was unique in its combination of an ostensibly postmortem phone call with "coded" psychokinetic answers to yes or no questions. However, oddly enough, while I was writing this review, I decided to take a break and go read for pleasure a book that had just come out a few days before: Mary Helen Hensley's Understanding Is the New Healing (2019). To my surprise, while blithely reading this book, I encountered another such case: that is, another ostensibly postmortem phone call with coded psychokinetic answers to yes or no questions (p. 127). This case began when a friend of Hensley's, Tanya, mentioned that she still had a hard time believing that her father, Sean, was gone, even many years after his death. Tanya and Hensley were together at the time, and they both saw the light in the room start flickering. They also saw both of Tanya's two cell phones begin to light up and flash on and off, though without ringing. Then a mechanical voice emerged from one of the phones, saying, "I am here ... Proud of you. ..." Hensley reports that the women then decided to use a pendulum in an attempt to prolong the communication. They asked to be shown which direction of swing would stand for yes and which for no, and they then proceeded to ask questions. "Sean ... is that you?" asked Tanya, and the pendulum began to swing emphatically in the yes direction. Hensley indicates that the conversation continued for at least a little while, and that the lights continued to flicker throughout. ${ }^{1}$

But let's get back to Kasprowicz's case. What does he himself make of his experience, and those of the other people whose accounts he's collected? For obvious reasons, he is intrigued by parallels between postmortem phone communications and poltergeist phenomena. Specifically, he is intrigued by a hypothesis often used to explain poltergeist phenomena: that they are created by the psychokinetic action of a living individual or individuals. In support of this as an explanation for ostensibly postmortem phone calls, Kasprowicz cites three cases (one taken from Rogo and Bayless and two from Cooper) 
in which people received telephone calls from living persons who had apparently had the intention or at least the strong motivation to place the calls in question but did not physically or consciously do so.

Along these same lines, I would add another case where a phone call apparently originated in a paranormal way from a living person but the primary motive for the communication nevertheless seemed to be on the side of the recipient, not the ostensible "caller." The ostensible caller in this case was author and anesthesiologist Jean-Jacques Charbonier, whose original account of the case is included in his book Les 7 bonnes raisons de croire à l'au-delà (Charbonier, 2012, pp. 164-166). I also describe this case in some detail in The Source and Significance of Coincidences (Rawlette, 2019, pp. 309-310). Charbonier's case suggests even more strongly than the cases from Rogo and Bayless and from Cooper that people may be capable of psychokinetically creating phone calls from those they have a strong desire to hear from. It clearly raises the possibility that living persons could use PK to create phone calls that have the appearance of coming from deceased individuals (though I should note that this is my own conclusion and not necessarily that of Charbonier).

Kasprowicz explores as well other phenomena often cited as providing evidence of communication from deceased persons: apparitions and electrical effects, for example, as well as mediumship. In each area, however, he finds that the evidence is ambiguous. Some cases seem to point toward the involvement of a deceased personality, especially when information unknown to the recipient of the ostensible communication is conveyed or when similar phenomena connected to the same deceased individual occur independently to multiple people in multiple locations at around the same time. And yet Kasprowicz finds that, as with telephone calls from the "dead," there is often some detail that tells against the phenomenon as the straightforward production of a deceased individual. For instance, he cites a reading he had with a medium. The reading was dazzlingly accurate at first, but the curious thing was that, though the medium seemed to be delivering communications from Kasprowicz's deceased grandmother, the "grandmother" communicating through the medium didn't do the one thing that Kasprowicz would have most expected her to do: wish him a happy birthday, since it was indeed his birthday, and she had 
never missed wishing him a happy birthday while she'd been alive.

Kasprowicz emphasizes two things that we must keep in mind as we seek to puzzle out the origins of ostensibly postmortem telephone calls. The first is that living people are able to create paranormal phenomena without the help of the dead. The second is that the mere fact that living people do sometimes create paranormal phenomena doesn't mean that they create all such phenomena; the dead could nevertheless create them as well.

In the final section of his book, Kasprowicz pursues his most theoretically interesting reflections. He considers that the bafflingly elusive quality of these phenomena may in fact be one of their defining characteristics and that the Trickster archetype may have a large role to play in their explanation. He discusses Carl Jung's appeal to the Trickster archetype as an explanation for poltergeist phenomena, as well as mentioning George Hansen's sociological exploration of the relationship between the Trickster and psi phenomena (Hansen, 2001).

The Trickster through the millennia and through many different cultures has been understood as both a messenger and a practical joker. The Trickster conveys the truth but always with some measure of play, humor, or absurdity-perhaps to take the edge off? Kasprowicz mentions that the Trickster may use the absurd as "protection," a way of covering his tracks. Some people who have encountered the Trickster have experiences so crazy that no one else will take them seriously. Their predicament is worsened by the tendency of these phenomena to flee scrutiny, disappearing when skeptics put them under the microscope. As Kasprowicz points out, more than one voice recording of a "message from the dead" has spontaneously erased itself, greatly contributing to the skepticism surrounding this phenomenon. But he suggests this may be precisely the Trickster's goal: to elude public investigation and systematization while nevertheless conveying important, private truths to those who come into contact with him. Kasprowicz notes that hiding and joking around are not the same as deception. He writes, "It could be that even when the Trickster is at work for example, the phenomenon is telling the truth" (p. 118)—that is, telling us that our deceased loved ones do live on.

It is also very interesting that Kasprowicz was first prompted to consider the role of the Trickster in postmortem phone communications 
because of a very specific experience he had involving one of the Trickster's most common representations in Western culture: the jester of the medieval and Renaissance royal courts. A few months after Kasprowicz experienced the strange phone calls featuring the hard breathing of a dog and rapped responses to his questions, Kasprowicz was driving on the highway when he fell asleep at the wheel. While asleep, he had a dream in which a court jester stepped out from behind a red curtain on a theater stage and, after looking at Kasprowicz for a moment, made a gesture with his hands indicating that Kasprowicz needed to turn the steering wheel to the right. Kasprowicz immediately opened his eyes and did so, and in this manner only barely avoided hitting the barrier in the median of the highway.

Kasprowicz notes that he hadn't had any recent contact with images of jesters before this experience; the image seemed to come to him out of nowhere. And, what was more, he says that the colors and sounds of the dream were unlike almost any other dream he has ever had in how very real they seemed. Kasprowicz considers the possibility that this dream — which quite plausibly saved his life-was also designed to give him a brief peek behind the curtain of the stage of life, revealing that the true actor at work in his paranormal experiences was the Trickster.

As for who-or what-the Trickster is exactly, Kasprowicz is clear that this archetypal being has strong links to the human unconscious. "Above all," he says, "it's a mechanism, an extraordinary mode of functioning of an autonomous part of our unconscious" (p. 107, my translation). That is, the Trickster is paradoxically both part of us and autonomous from us at the same time. Kasprowicz also seems to be attracted to the conclusion that philosopher Philippe Solal expresses in his Afterword to Kasprowicz's book, which is that it's the Soul or the Higher Self that is behind ostensible messages from the dead. Solal writes:

As the soul is the interface between oneself and the invisible world of the dead, it is the soul that addresses these messages to us, even if it's not impossible that it is sometimes serving as a messenger for the dead themselves, acting as their spokesperson. (p. 129, my translation) 
However, it is precisely because Kasprowicz's book Des coups de fil de l'AU-DELA? doesn't expound any firm thesis that I highly recommend it to anyone interested in ostensible postmortem communication or indeed in any sort of psychokinetic phenomenon. Kasprowicz does not shy away from the difficult questions and offers no easy answers. His goal is to explore the phenomenon of telephone communication with the dead in its full puzzling variety, and he does this methodically and with elegance, while also offering us, in his discussions of the Trickster, an important lead that I believe all future theorizing on the subject would be wise to consider.

\section{NOTE}

- I have also discovered, since beginning to write this review, that the first iteration of the SoulPhone ${ }^{\mathrm{TM}}$ technology being developed by Gary E. Schwartz and the SoulPhone Foundation employs coded psychokinetic responses to yes or no questions. However, since the technology does not appear at this point to involve the use of ordinary telephones, I would not consider instances of its use examples of postmortem telephone calls so much as a technological variation on the more familiar techniques of physical mediumship. For more information, see https://www.thesoulphonefoundation.org/the-soulphone-project

\section{REFERENCES}

Charbonier, J.-J. (2012). Les 7 bonnes raisons de croire à l'au-delà. Guy Trédaniel, J'ai Lu. Cooper, C. E. (2012). Telephone calls from the dead. Tricorn Books.

Hansen, G. P. (2001). The trickster and the paranormal. Xlibris.

Hensley, M. H. (2019). Understanding is the new healing: Miraculous recoveries from physical and emotional trauma. Lisa Hagan Books, Love Never Dies imprint.

Rawlette, S. H. (2019). The source and significance of coincidences: A hard look at the astonishing evidence. Sharon Hewitt Rawlette.

Rogo, D. S., \& Bayless, R. (1979). Phone calls from the dead: The results of a two-year investigation into an incredible phenomenon. Prentice-Hall. 